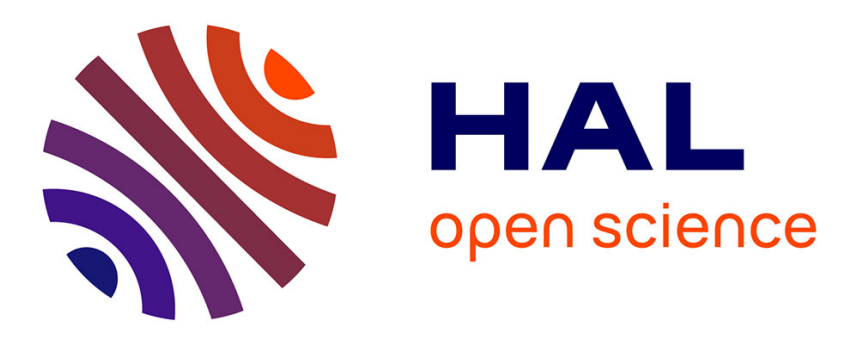

\title{
Un grand détecteur E.M. à haute réjection des pions
}

D. Bollini, A. Buhler-Broglin, P. Dalpiaz, T. Massam, F. Navach, F.L. Navarria, M. A. Schneegans, A. Zichichi

\section{To cite this version:}

D. Bollini, A. Buhler-Broglin, P. Dalpiaz, T. Massam, F. Navach, et al.. Un grand détecteur E.M. à haute réjection des pions. Revue de Physique Appliquée, 1969, 4 (2), pp.108-110. 10.1051/rphysap:0196900402010801 . jpa-00243167

\section{HAL Id: jpa-00243167 https://hal.science/jpa-00243167}

Submitted on 1 Jan 1969

HAL is a multi-disciplinary open access archive for the deposit and dissemination of scientific research documents, whether they are published or not. The documents may come from teaching and research institutions in France or abroad, or from public or private research centers.
L'archive ouverte pluridisciplinaire HAL, est destinée au dépôt et à la diffusion de documents scientifiques de niveau recherche, publiés ou non, émanant des établissements d'enseignement et de recherche français ou étrangers, des laboratoires publics ou privés. 


\title{
UN GRAND DÉTEGTEUR E.M. A HAUTE RÉJEGTION DES PIONS
}

\author{
D. BOLLINI, A. BUHLER-BROGLIN, P. DALPIAZ, T. MASSAM, F. NAVAGH, \\ F. L. NAVARRIA, M. A. SGHNEEGANS et A. ZIGHIGHI, \\ C.E.R.N., Geneva, Switzerland, \\ Istituto di Fisica dell'Università di Bologna, Italie, \\ Istituto Nazionale di Fisica Nucleare, Sezione di Bologna, Italie, \\ Centre de Recherches Nucléaires, Strasbourg, France.
}

Résumé. - Un grand détecteur de gerbes électromagnétiques comprenant neuf couches - chacune faite de plomb, d'une chambre à étincelles et d'un compteur à scintillations - est décrit. La réjection contre les pions est de $6 \times 10^{-4}$, l'efficacité de détection des électrons varie de $68 \%$ à $80 \%$ et la résolution en énergie de $15 \%$ à $10 \%$ dans la région d'énergie de $0,4 \mathrm{GeV}$ à $1,1 \mathrm{GeV}$.

Abstract. - A large electromagnetic shower detector which consists of nine layers, each one made of lead, spark-chamber and scintillation counter, is described. The rejection power against pions is of $6 \times 10^{-4}$, the efficiency for electron detection varies from $68 \%$ to $80 \%$ and the energy resolution from $15{ }_{\%}^{\circ}$ to $10_{\%}^{\circ}$ in the energy range $0.4 \mathrm{GeV}$ to $1.1 \mathrm{GeV}$.

I. Introduction. - En 1964, la collaboration Bologne-G.E.R.N.-Strasbourg a construit et étudié un détecteur [1] constitué de couches successives de plomb, de chambres à étincelles et de scintillateurs en « sandwich », capable de distinguer les électrons des pions à des énergies allant de 1,1 à $2,5 \mathrm{GeV}$. Le détecteur décrit ici est une version agrandie, réalisée en 1967, capable de détecter des électrons ou des 


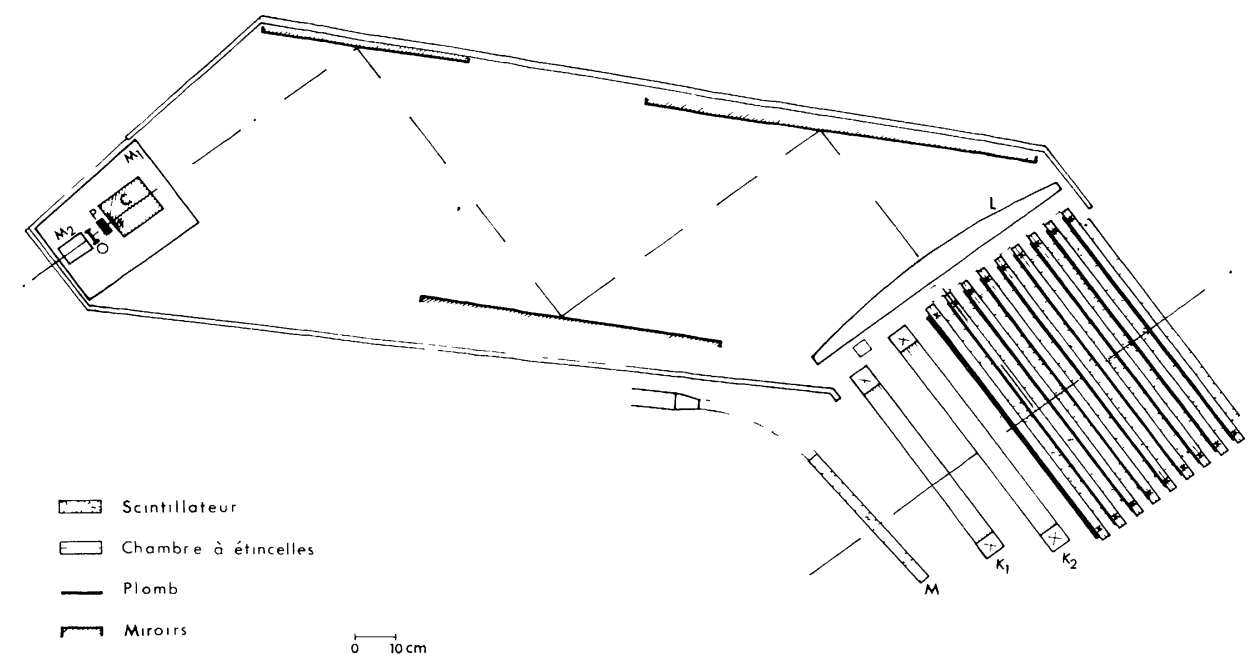

FIG. 1. - Section verticale du détecteur : devant les neuf couches du "sandwich ", on voit les chambres à étincelles à feuilles minces $\mathrm{K}_{1}$ et $\mathrm{K}_{2}$ et le compteur $\mathrm{M}$. La lentille $\mathrm{L}$, les grands miroirs, les miroirs à $45^{\circ} \mathrm{M}_{1}$ et $\mathrm{M}_{2}$, l'objectif $\mathrm{O}$ amènent l'image des chambres sur le film $\mathrm{F}$. Les croix sur les chambres à étincelles sont des marques fiducielles.

rayons $\gamma$ à des énergies allant de quelques centaines de $\mathrm{MeV}$ à plusieurs $\mathrm{GeV}$ en présence d'un important fond de pions. Ce détecteur combine la méthode électronique utilisant les impulsions produites par des compteurs à scintillation pour réaliser une discrimination électron-pion et la méthode optique qui consiste à visualiser les gerbes dans des chambres à étincelles et permet ainsi d'améliorer la réjection des pions. Deux tels détecteurs ont été utilisés dans plusieurs expériences $[2,3,4]$.

II. Description. - La figure 1 représente une section verticale du détecteur. Il consiste essentiellement en un « sandwich » compact de neuf couches triples comprenant chacune une plaque de plomb, une chambre à étincelles et un compteur à scintillation. Les dimensions du parallélépipède sensible sont : $60 \times 120 \mathrm{~cm}^{2}$ de surface et $50 \mathrm{~cm}$ de profondeur.

L'épaisseur totale de plomb correspond à 8,6 longueurs de radiation. La première plaque a $1 \mathrm{~cm}$ d'épaisseur assurant, avec une probabilité supérieure à $80 \%$, la multiplication de l'électron incident.

Les neuf chambres à étincelles identiques ont chacune deux intervalles de $0,85 \mathrm{~cm}$ de large et leurs dimensions sont : $70 \times 130 \times 2 \mathrm{~cm}^{3}$. Leurs plaques sont des feuilles d'aluminium de $1 \mathrm{~mm}$ d'épaisseur tenues par un cadre de plexiglas. Une barre centrale de $0,7 \mathrm{~cm}$ de large sépare les chambres en deux moitiés optiquement indépendantes. Les chambres sont remplies d'hénogal $(30 \% \mathrm{He}, 70 \% \mathrm{Ne})$ et une haute tension de $9 \mathrm{kV}$ est appliquée sur le plateau central. Un champ de balayage de $30 \mathrm{~V}$ donne $95 \%$ d'efficacité pour un temps de sensibilité de $1 \mu s$.

Les neuf compteurs à scintillation identiques se composent chacun d'une plaque de scintillateur plas- tique de $60 \times 120 \times 2,5 \mathrm{~cm}^{3}$, vue par 4 photomultiplicateurs 56 AVP à travers deux guides de lumière rectangulaires de $60 \times 65 \times 2,5 \mathrm{~cm}^{3}$ prolongeant le scintillateur à ses deux extrémités plus étroites.

On peut voir ( fig. 1), en avant du « sandwich », les deux chambres cinématiques à feuilles minces $(25 \mu \mathrm{m})$, comportant chacune 6 intervalles de $0,8 \mathrm{~cm}$ et permettant de reconstruire la direction des particules chargées incidentes avec une précision de \pm 10 . Devant ces chambres, un compteur à scintillation $(\mathbf{M})$ garantit une particule incidente chargée et réduit la multiplicité de ces particules. Lorsque le détecteur est utilisé comme spectromètre de rayons $\gamma$, un convertisseur de plomb est ajouté.

Le système optique permettant de photographier avec une seule caméra les deux moitiés optiquement indépendantes des chambres est visible sur la figure 1 .

Chaque moitié du système transmet l'image de deux vues orthogonales des chambres.

La logique électronique adoptée après différentes études consiste à demander une certaine multiplication dans la première plaque de plomb ainsi qu'une énergie totale laissée par la particule dans le détecteur supérieure à une certaine valeur. L'impulsion discriminée du premier compteur ainsi que la somme linéaire discriminée des neuf compteurs sont envoyées dans la coïncidence finale qui déclenche la logique des chambres à étincelles.

III. Calibrations. - Calibrer le détecteur consiste à le placer dans un faisceau direct d'électrons ou de pions et à mesurer sa réponse en hauteur d'impulsion et en efficacité, à différents moments de la particule incidente. On peut ainsi déterminer les conditions électroniques pour réaliser le meilleur compromis entre 


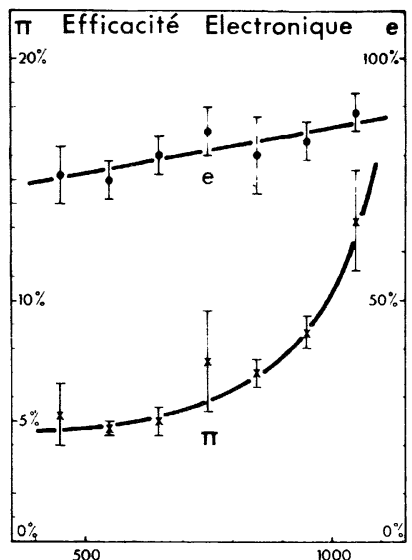

a)

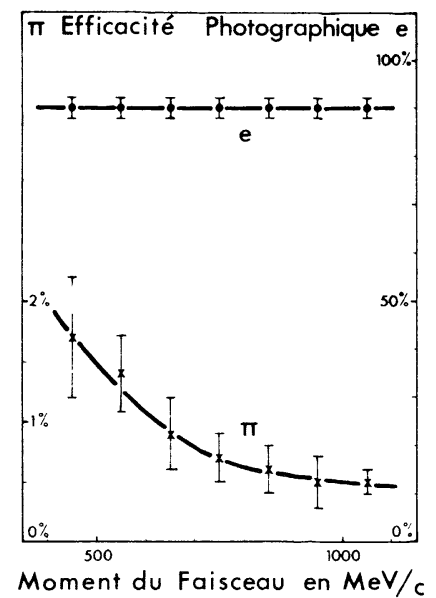

b)

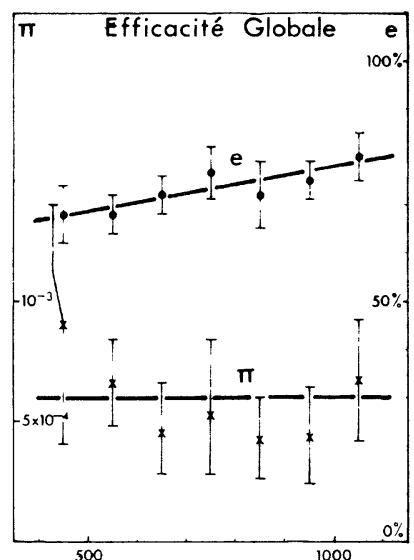

c)

FIG. 2. - Efficacités de détection "électronique ", " photographique " et globale , pour les électrons et les pions en fonction du moment de la particule incidente.

haute efficacité de détection pour les électrons et bonne réjection des pions. Ceci est la «calibration électronique ». Dans une deuxième étape, on prend des photos des chambres à étincelles dans les conditions électroniques déterminées de manière à définir des critères pour une réjection supplémentaire des pions. C'est la «calibration photographique ». Ces calibrations ont été effectuées en de nombreux points de la surface du détecteur de manière à connaître l'efficacité moyenne de détection et à étudier son uniformité et les effets de bord.

IV. Performances. - Les résultats des « calibrations électroniques » et des «calibrations photographiques » sont représentés respectivement sur les figures $2 \mathrm{a}$ et $2 \mathrm{~b}$ en fonction du moment de la particule incidente.
La figure 2 c montre les efficacités totales pour les pions et les électrons, obtenues en multipliant les efficacités « électroniques » et « photographiques » à différents moments. Les valeurs citées sont des moyennes sur différentes positions d'impact du faisceau et sur différentes périodes de temps des efficacités de détection et les erreurs représentées contiennent les fluctuations avec ces deux facteurs. On voit que l'efficacité de réjection totale pour les pions $\left(\sim 5 \times 10^{-4}\right)$ ne varie pas sensiblement avec le moment, pendant que l'efficacité pour les électrons augmente de $68 \%$ à $450 \mathrm{MeV} / \mathrm{c}$ à $80 \%$ à $1050 \mathrm{MeV} / \mathrm{c}$.

La résolution en énergie du détecteur, obtenue en combinant l'information des hauteurs d'impulsions et celle du nombre d'étincelles présentes dans la gerbe, est de $15 \%$ à $400 \mathrm{MeV}$ et de $10 \%$ à $1100 \mathrm{MeV}$.

\section{BIBLIOGRAPHIE}

[1] Massam (T.) et al., Nuovo Cim., 1965, 39, 464.

[2] Bollini (D.) et al., Nuovo Cim., 1968, 56 A, 1173.
[3] Bol,ini (D.) et al., Nuovo Cim., 1968, 57 A, 404.

[4] Bol.LINI (D.) et al., Nuovo Cim., 1968, 58 A, 289. 